\title{
A Tale of Two Transitions: Unraveling Two Distinct Polymorph Transition Mechanisms in One n-Type Single Crystal for Dynamic Electronics
}

Daniel William Daviesa, Sang Kyu Park, Stephen B. Shiring ${ }^{b}$, Hyunjoong Chung ${ }^{a}$, Prapti Kaflea, Dafei Yuan $^{c}$, Joseph W. Strzalka ${ }^{d}$, Ralph Weber ${ }^{\mathrm{e}}$, Xiaozhang Zhuc, Brett M. Savoie ${ }^{\mathrm{b}}$, Ying Diao*a,f

\footnotetext{
aDepartment of Chemical and Biomolecular Engineering, University of Illinois at Urbana-Champaign, 600 South Mathews Avenue, Urbana, IL 61801, USA. bDavidson School of Chemical Engineering, Purdue University, 480 W Stadium Ave, West Lafayette, IN
47907
}

'Beijing National Laboratory for Molecular Sciences, CAS Key Laboratory of Organic Solids, Institute of Chemistry, Chinese Academy of Sciences, Beijing 100190, P. R. China

${ }^{d} X-$ Ray Science Division, Argonne National Laboratory, Argonne, IL 60439, USA

'Bruker BioSpin Corp., 15 Fortune Drive, Billerica, MA 01821, United States

fBeckman Institute for Advanced Science and Technology 405 N. Mathews Ave. M/C 251, Urbana, IL 61801

Note: current address of Dr. Sang Kyu Park - Institute of Advanced Composite Materials, Korea Institute of Science and Technology, Joellabuk-do, 55324, South Korea

Current address of Dr. Dafei Yuan - Materials Department, University of California, Santa Barbara, Engr II, UCSB Engineering II, Santa Barbara, California, 93117, United States

For correspondence: yingdiao@illinois.edu 


\begin{abstract}
Cooperativity is used by living systems to circumvent energetic and entropic barriers to yield highly efficient molecular processes. Cooperative structural transitions involve the simultaneous, concerted displacement of molecules in a crystalline material, in stark contrast to the more typical molecule-bymolecule nucleation and growth mechanism often breaking the single crystallinity. Cooperative transitions have acquired much attention in the research community for their low transition barriers, ultrafast kinetics, and structural reversibility. On the other hand, cooperative transitions are rarely observed in molecular crystals and the molecular origin is not well understood. Single crystals of 2dimensional quinoidal terthiophene (2DQTT-o-B), a high-performance n-type organic semiconductor, demonstrate two thermally-activated, reversible phase transitions with one exhibiting a cooperative mechanism and the second exhibiting a nucleation and growth mechanism. In situ microscopy, single crystal and grazing incidence X-ray diffraction (GIXD), along with Raman spectroscopy suggest a reorientation of the alkyl side chains results in a cooperative transition behavior. On the other hand, the nucleation and growth transition is coincident with both side chain melting and the emergence of new spin-spin interactions between conjugated cores, confirmed through in situ electron paramagnetic resonance spectroscopy (EPR). This is the first observation of biradical interactions directly initiating a structural transition. Through studying these fundamental mechanisms, we establish alkyl chain conformation and disorder as integral to rationally controlling these polymorphic behaviors for novel electronic applications.
\end{abstract}

\title{
Introduction
}

A class of emergent phenomenon ${ }^{1}$, known as cooperativity, grants the bypassing of large energy barriers in a wide variety of systems, ranging from spin flipping in magnetic materials to protein folding ${ }^{2-5}$. In solid state phase transitions, cooperative mechanisms occur by a concerted displacement of molecules with each molecule retaining its nearest neighbors. This results in ultrafast kinetics and lower energy barriers, relative to typical nucleation and growth type mechanisms, which occur molecule by molecule. The displacive nature of cooperativity, well documented in inorganic martensitic transitions, results in unique behaviors, such as reversible shape changes in the crystal leading to thermosalient motion $^{6-9}$ and shape memory ${ }^{7,10}$. A key distinction from nucleation and growth rises from interactions with defects within the crystal, which results in avalanche behavior due to pinning of the phase boundary ${ }^{4,11-14}$. While cooperative behavior has been well documented in metal alloy based martensitic transitions, it has rarely been reported in molecular crystals. As such, the fundamental origin of cooperativity in organic crystals is not well understood and the molecular design inducing this behavior is still unknown.

Recently, cooperative transitions have been observed in key organic semiconductor molecules such as 6,13-bis(triisopropylsilylethnyl) pentacene (TIPS-P), among other p-type semiconductors ${ }^{9,15,16}$. Cooperativity in TIPS-P led to fast switching of the electronics ${ }^{17}$ coupled with large recoverable crystal shape change ${ }^{15}$, resulting in super- and ferro-elastic mechanical effects ${ }^{18}$. In the case of TIPS-P, the rotation of the TIPS groups played a key role in the observed cooperative phenomena ${ }^{16,19}$. Besides 
dynamic rotation, other systems have shown the importance of molecular tilting and order-disorder mechanisms to resolve cooperative behaviors ${ }^{20-22}$. The switching of electronic properties coupled with the rapid mechanical effects of cooperative phase transitions presents a wide design space for novel organic electronics such as thermally activated actuators and highly flexible devices ${ }^{9,23}$.

However, studies involving structural transitions in n-type counterparts (necessary for full logic design) have severely lagged behind. $\mathrm{N}$-type semiconductors require relatively low lying LUMO levels for efficient electron transport, which are difficult to stabilize under ambient conditions ${ }^{24-27}$. One route to design stable n-type molecules involves forming a quinoidal structure using strong electron withdrawing groups such as cyano groups and attaching long alkyl chains to the core. The cyano groups break the aromaticity of the conjugated core and planarize the molecule by stabilizing the quinoidal resonance structure that exhibits double bonds between the rings. On the other hand, alkyl chains are widely used to impart solution processability and helps prevent reactions with water and oxygen in the solid state $e^{24,28}$. At the same time, these quinoidal cores stabilize an exotic biradical ground state that exists in equilibrium with the quinoidal form ${ }^{24,28-30}$. The presence of biradicals results in interesting spin-spin interactions ${ }^{31,32}$ between the conjugated cores and, in extreme cases, results in formation of "pancake" bonding ${ }^{33}$. These unique interactions are also tunable through slight changes in environment (concentration, temperature etc.). Along with intermolecular interactions, biradical formation appears to have self-doping effects capable of tuning the charge carrier densities and the resulting electronic properties ${ }^{12,28,34}$. While both the quinoidal core and alkyl chains are key to engineering n-type semiconductor molecules, the effect on structural transitions is still unexplored.

In this work, we report an intriguing coexistence of both cooperative and nucleation and growth type mechanisms during polymorphic transitions in single crystals of 2DQTT-o-B, one of the highest performing $\mathrm{n}$-type organic semiconductors to date ${ }^{24,35}$. The I-II phase transition, which exhibits cooperativity, is accompanied by a significant conformational change of the alkyl side chains, showing the importance of side chain flexibility in accessing cooperative phenomena. In stark contrast, the II-III transition shows a nucleation and growth mechanism driven by an increase in core interactions based on biradical formation in combination with melting of the alkyl side chains. This is the first time that both cooperative and noncooperative transitions are reported in a single system. Further, the phenomenon of biradical interactions directly inducing a polymorphic phase transition has not been reported before. Finally, we demonstrate the use of cooperative behavior through a thermally actuated switching device, a functionality unavailable to a nucleation and growth type mechanisms.

\section{Results and discussion}

Polymorph Transition Behaviors. Crystals of 2DQTT-o-B (Figure 1a) were either grown via drop casting from a 1:1 para-dichlorobenzene and decane mixture or slow evaporation from a 1:1 dichloromethane and ethyl acetate mixture to form both small $(50-300 \mu \mathrm{m})$ and large $(1-3 \mathrm{~mm})$ crystals, respectively (Methods Section). Single crystal X-ray diffraction showed crystals of 2DQTT-o-B packed in a $\mathrm{C}_{2 / \mathrm{c}}$ unit cell with 1-D $\pi$-stacks which was designated polymorph $\mathrm{I}^{35}$. Based on the BFDH morphology (Figure S1), we observe the $\pi-\pi$ stacking occurs along the long axis of the crystal (Figure 1 $\mathbf{b}$ ). These $\pi$-stacks then pack into layers separated by the alkyl side chains (Figure 1c), which results in the thin needle like crystals observed in polarized optical microscopy (POM). We set to investigate two thermally induced structural transitions in single crystals of 2DQTT-o-B using polarized optical microscopy, where we heated and 
cooled crystals at a constant $5{ }^{\circ} \mathrm{C} \mathrm{min}^{-1}$. We observed two reversible phase transitions designated the I-II transition at $164{ }^{\circ} \mathrm{C}$ and II-III at $223{ }^{\circ} \mathrm{C}$. The phase transitions were accompanied by modulation of the brightness and color, reflecting changes in the refractive index of the material (Figure 1d,e, Movies 1-2,34). We observed distinct transition behavior suggesting a cooperative transition mechanism for the I-II transition as opposed to a nucleation and growth mechanism during the II-III transition. Below we discuss evidence from in situ POM in detail and this inference is further supported by in situ GIXD, Raman spectroscopy and EPR spectroscopy discussed later.

Using python-based image analysis, we were able to select individual crystals and track the progression of the phase transition by calculating an average intensity for each frame. The I-II transition proceeded remarkably fast upon heating (Figure 1d, Movie S1,2). Across many crystals, the heating transition typically occurred within one frame, less than 0.1 second, providing a lower bound for the propagation speed of at least $2000 \mu \mathrm{m} \mathrm{s}^{-1}$, implying a cooperative-type mechanism. While difficult to capture under normal circumstances due to the speed, this rapid transition is accompanied by a well-defined phase front propagating through the crystal, distinct from typical diffuse phase boundaries under nucleation and growth mechanisms. Initiation of such phase front typically occurred at the crystal tips, or at cracks within the crystal. Even when the transition initiated at both tips simultaneously, the resulting crystal was a single domain after transition, suggesting a close orientational relationship between the two phases. This is consistent with our earlier work by Chung and Diao et. al. ${ }^{36}$ which showed defects facilitated phase boundary initiation in similar cooperative transitions of ditBu-BTBT followed by cooperative propagation.

During cooling the sharp phase boundary becomes much more evident due to avalanche behavior, where the phase boundary became pinned during transition (Figure 1d, Movie S2). These avalanches follow behavior seen in inorganic martensitic transitions, where defects in the lattice, such as dislocations, create a local free energy minimum and corresponding energy barrier ${ }^{11,14,37}$. The resulting rough free energy landscape causes the boundary to move in a series of jerks and requires thermal fluctuations to jump over the defect barriers. As mentioned, the avalanches were found during cooling, which is clearly observed as plateaus in the crystal intensity during the temperature changes (Figure $\mathbf{1 f}, \mathbf{g}$ ). This suggests the initial heating transition may be resulting in an increasing number of defect sites within the crystal that cause avalanches in subsequent cycles. However, despite taking several seconds, up to minutes, to become unpinned, this typically does not change the speed of the transition as each avalanche still occurs within less than one frame of the video (Figure 19).

Moreover, the direction of the phase boundary is only observable during these pinning events. In these cases, we observe two main crystallographic directions along which the boundary forms: the (010) and (025) planes both of which are connected to the $\pi$-stacking direction. The (010) direction lies perpendicular to the 1-D $\pi$-stacks, suggesting the cooperative behavior is transmitted down the $\pi$-stacks, similar to a domino effect (Figure 1i). On the other hand, the (025) plane parallels the conjugated core of the molecule and was indexed to the $\pi-\pi$ stacking peak in grazing incidence X-ray diffraction ${ }^{35}$. The crosshatch structure of the $\pi$-stacking results in two mirrored directions within the crystal, following the two-phase boundary directions (Figure S3a-c). This would suggest a cooperative behavior is transmitted along the 1-D $\pi$-stack and is inhibited by dislocations present in those stacks.

In contrast, the II-III phase transition (Figure 1e, Movies S3,4) showed nucleation at several points within the crystal and transformed over several minutes, with polymorph III increasing in brightness under polarized microscopy. The II-III transition exhibited no clear phase boundary, instead we observed a 
diffuse spreading of the intensely bright polymorph III. The most common nucleation points are near the edges of the crystal, though cracks and other defects will act as nucleation spots (Figure 1e) as well. By tracking the intensity change over temperature, we observe a quite smooth change, with no avalanche like behavior (Figure 1f,h). Upon cooling, the reverse transition began at a temperature below that of the I-II transition, exhibiting a hysteresis of $100^{\circ} \mathrm{C}$, an order of magnitude greater than the I-II transition which showed a hysteresis of only $17 \pm 5{ }^{\circ} \mathrm{C}$, confirming a much larger energy barrier for nucleation and growth mechanism. This matches with observations from DSC using powder samples, showing a hysteresis for the I-II and II-III transitions of $6^{\circ} \mathrm{C}$ and $75{ }^{\circ} \mathrm{C}$, respectively ${ }^{35}$. In single crystals, the hysteresis for the II-III transition varied substantially and, in some cases, the III-I transition did not occur during constant cooling, resulting in the kinetic trapping of polymorph III at room temperature. This suggested a nucleation and growth behavior, shown in Figure $\mathbf{1} \mathbf{j}$, where nucleation occurred at edges or defects and diffusively spread through the crystal. 
a)

d)

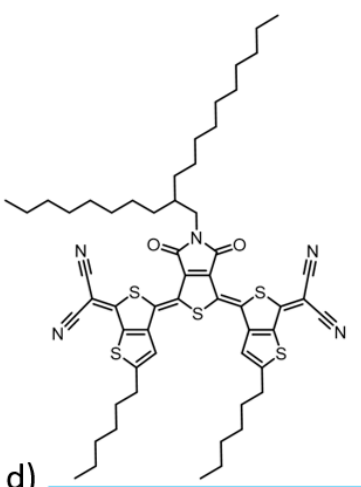

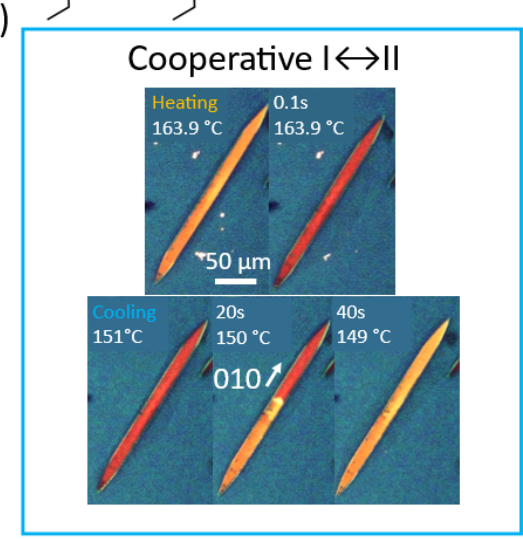

f)

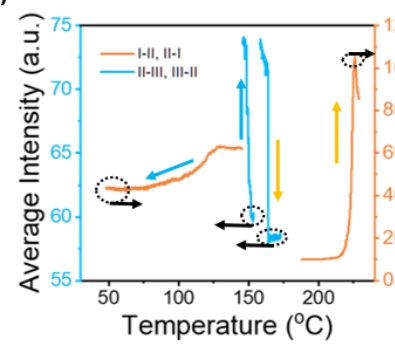

b)

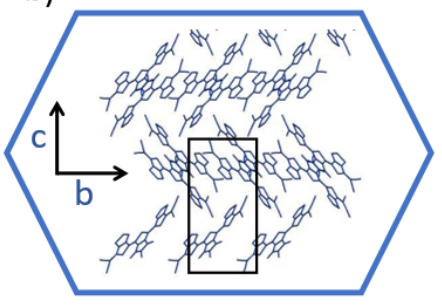

e)

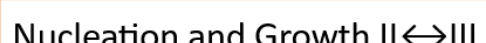

Nucleation and Growth $\| \leftrightarrow I I I$

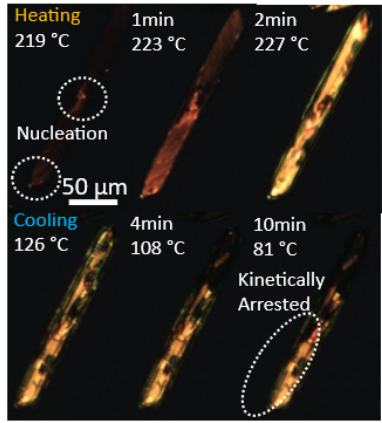

h)

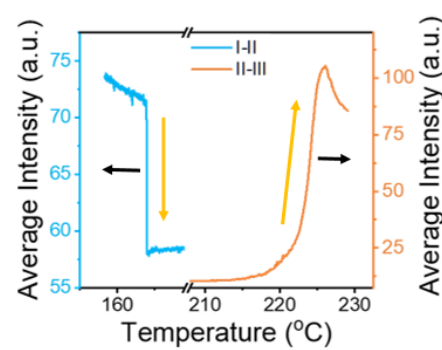

II $\leftrightarrow$ II g)

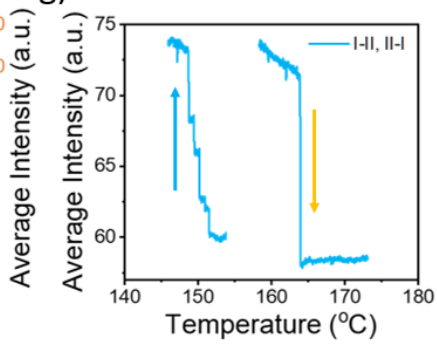

c)

a

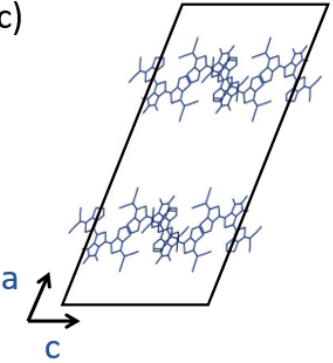

i) Initiation

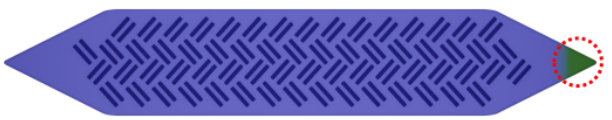

Propagation

j) Nucleation
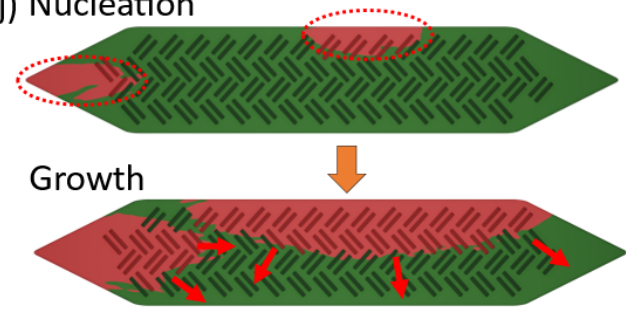

Figure 1. Comparison of single crystal transition kinetics. (a) 2DQTT-o-B molecular structure. Crystal packing of polymorph I in the crystal viewed from (b) the top of the crystal (down $a^{*}$ ) and (c) along the $b$ direction. The crystal shape is outlined in blue and the unit cell is outlined in black. In situ POM showing the I-II (d) and II-III (e). (f) By tracking the transition progress via intensity changes of the crystals, we observe the avalanche behavior of the I-II transition $(\mathrm{g})$ and see the stark difference in kinetics $(\mathrm{h})$ between the I-II and II-III heating transitions. The dark blue and red lines indicate the I-II and II-III transitions respectively and the yellow and blue arrows indicate the heating and cooling transitions respectively. Black arrows indicate the axis for each line. Schematic showing the typical modes for initiation and propagation of the (i) I-II and (j) II-III transition. 
Thermosalient behavior. Upon undergoing the I-II transition, we found the crystal decreased in length by $3.6 \pm 1.3 \%$ on average (Figure $\mathbf{2 a}$, Table S1). This change in length was matched quite closely with the $2.9 \%$ decrease in the b-direction of the unit cells obtained through fitting the thin film GIXD diffraction patterns (Figure $\mathbf{2 b}$ ), as we previously reported ${ }^{35}$. This shape change led to cracking of pinned small crystals of $\sim 100 \mu \mathrm{m}$ in size (Figure S2, Movie S5), which occurs due to the buildup of strain at the phase front caused by the change in shape coupled with crystal-substrate interactions that act to prevent the shape change. In an extreme case, we observed a crystal embedded in a surrounding film that formed during the drying process, which cracked upon transition. This case also resulted in the formation of "bright bands" along the phase boundary, visible in Figure S4, which would be consistent with strain induced birefringence we expect from a crystal attempting the I-II transition while trapped in a solid film. On the other hand, for unpinned large crystals $(1-3 \mathrm{~mm})$, we observed a pronounced thermosalient behavior during the I-II transition (Figure 2c, Movie S6). While the crystal will bend during the I-II transition, reversing the transition will not recover original shape, implying a plastic deformation. This may be a result of strain buildup at the phase boundary causing the $\pi$-stacks within the crystal to irreversibly slip along the greasy alkyl chain layers.

In the II-III transition, however, we observed a slight expansion to the crystal without thermosalient behavior (Figure $\mathbf{2 d}$ ). The change did not conform to the unit cell changes, as polymorph III exhibits a vastly different symmetry and unit cell relative to polymorph II (Figure 2e) and results from thermal expansion during heating. This reconstructive transition also leads to the formation of multiple grain boundaries within the crystal, breaking single crystallinity (Figure 2f). Formation of these domains mitigate strain build up preventing any thermosalient motion from occurring due to the crystal expansion. These observations are consistent with the understanding that the nucleation and growth mechanism does not incur shape change owing to a single-to-polycrystalline transition ${ }^{38}$. This stands in contrast to the cooperative martensitic transition that preserves single crystallinity and thus exhibits shape reversibility corresponding to the unit cell change. 


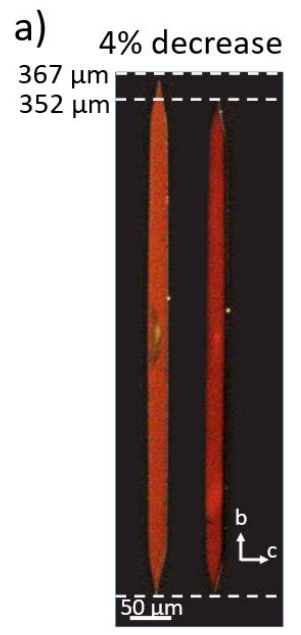

b)

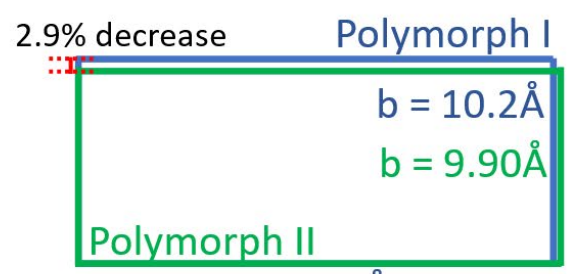

$\mathrm{c}=24.2 \AA$

$c=24.5 \AA$ d)

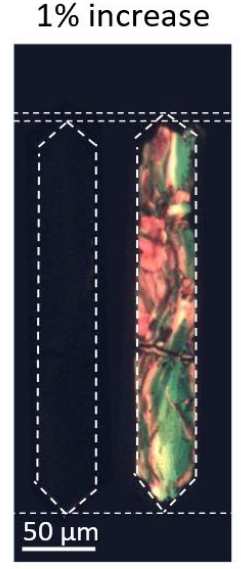

e)

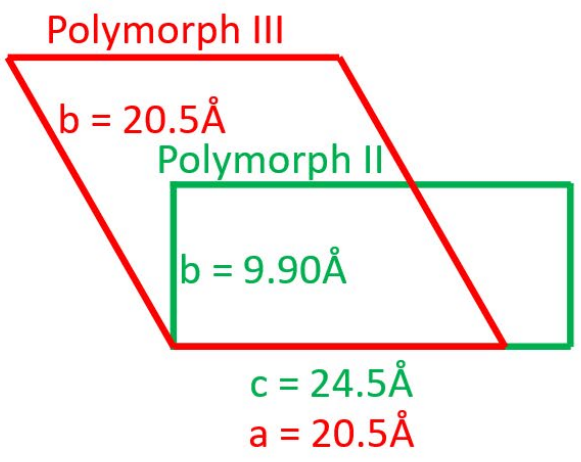

c)
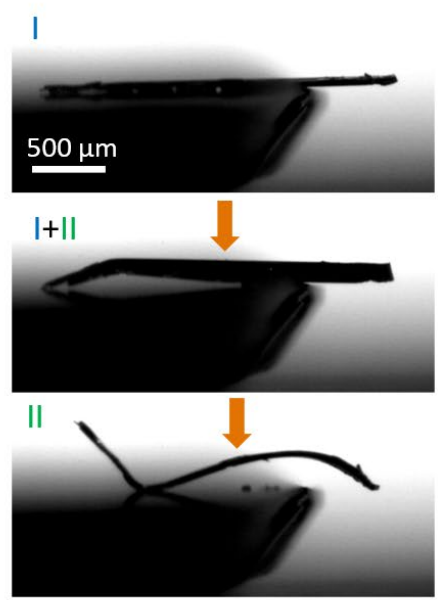

f)

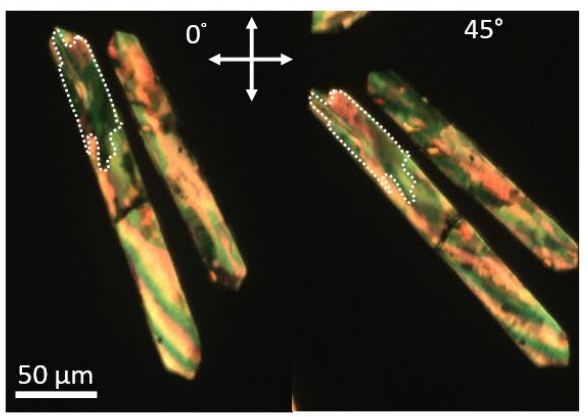

Figure 2. Shape change and thermosalient behavior. (a) Crystal shape change associated with the I-II transition and (b) associated in plane unit cell change accounting for the change in crystal length along the $b$ axis of the unit cell (unit cell drew in the same orientation as the crystal in (a)). (c) The resulting thermosalient effect in larger crystals caused by the shape change. (d) Crystal expansion observed in the II-III transition, compared to (e) the unit cell changes, where the unit cell is shown in the same orientation as the crystal in (d)), and observation of ( $f$ ) birefringence showing multiple domain formation under polarized optical microscopy.

Molecular Origin of Transition Mechanisms. Uncovering the origins of the transition behaviors requires understanding the molecular and structural modifications during the phase transitions. To that end, we performed in situ grazing incidence X-ray diffraction (GIXD) during thermally triggered polymorph transitions. A GIXD video of the I-II transition was obtained by continuous heating at $5{ }^{\circ} \mathrm{C} \mathrm{min}^{-1}$ and capturing the diffraction pattern every 5 seconds (Movie S7). The unit cells for polymorphs I and II were extracted from the GIXD patterns via a regression fitting the diffraction pattern as discussed in previous work $^{35}$. This fitting for polymorph I also matched the single crystal unit cell obtained at room temperature ${ }^{35}$. During the I-II transition, we observed minor changes to the unit cell, showing peak movements in the $q_{z}$ direction while keeping the Bragg rods in place and maintaining the monoclinic symmetry (Figure $\mathbf{3 a}, \mathbf{b}$ ). The largest extent of peak shift in the $q_{z}$ position occurred right before the I-II 
polymorph transition, similar to pretransition behavior observed by Panda and Naumov et. al. ${ }^{8,39}$ (Figure $3 \mathbf{c}, \mathbf{d})$. This pretransition behavior is typical of pretransition regions of critical phenomena ${ }^{40}$, such as in the lattice fluctuations necessary to drive the avalanche behavior ${ }^{41}$. Comparing the fitted unit cells for polymorphs I and II (Figure 3a), there is a significant increase in the $\beta$-angle during the I-II transition, consistent with the observed out of plane shift. By comparing single crystal X-ray diffraction with GIXD for polymorph I, we showed this angle reflects the molecular tilt with respect to the substrate, measured to be $66^{\circ}$ (Figure S5), commensurate with the complement of the $\beta$-angle, measured to be $67^{\circ 35}$. During the I-II transition, the $\beta$-angle changed from $113^{\circ}$ to nearly $90^{\circ}$, suggesting the molecules standing straight up from a more tilted position, like reverse dominos.

To elucidate the driving force behind this change in tilt, we turned to in situ Raman spectroscopy to provide insight into the molecular changes occurring in the I-II transition (Figure 3d,e). Raman has shown to be a powerful tool for tracking polymorphic transitions and understanding the molecular changes that occur as a result of these transitions ${ }^{15,42,43}$. Under Raman spectroscopy crystals exhibit both low frequency peaks related to the intermolecular phonon vibrations, sensitive to the crystal structure $<$ $\left.500 \mathrm{~cm}^{-1}\right)$, as well as intramolecular vibrations related to the conformation and chemical environment of the molecule $\left(900-1800 \mathrm{~cm}^{-1}\right)$. In the phonon region, we observe two key peaks which are associated with lattice vibrational modes, indicated with black arrows in Figure 3f. We observe a ratio change between these two peaks, as the lowest peak increases in intensity during the I-II transition (Figure $\mathbf{3 f}$ ). This is a small effect, indicating modest changes to the lattice packing consistent with our observation of changing molecular tilt from GIXD. Phonon intensity modulation, as opposed to peak shifts or splitting has been shown to be typical in well studied martensitic type cooperative transitions ${ }^{44,45}$. On the other hand, in the intramolecular vibration region (900-1800 $\mathrm{cm}^{-1}$ ), we observed significant changes to the intensity of the peak at $1409 \mathrm{~cm}^{-1}$ relative to the most intense peak at $1387 \mathrm{~cm}^{-1}$ indicated by the red and blue arrows in Figure $\mathbf{3 g}$, respectively and illustrated in Figure $\mathbf{3 e}$. Based on a combination of DFT calculations (Figure S6b) and literature, we assigned the peak at $1387 \mathrm{~cm}^{-1}$ to $C=C$ stretching along the backbone, corresponding to the effective conjugation coordinate (ECC) mode for the quinoidal form ${ }^{46-48}$ which we denote as $\mathrm{V}(\mathrm{C}=\mathrm{C})_{\mathrm{ECC}, \mathrm{Q}}$ (Figure S6c, Movie S8,9); the peak at $1522 \mathrm{~cm}^{-1}$ was assigned to other $\mathrm{C}=\mathrm{C}$ stretching modes in the backbone (Figure s6e, Movie s10); the peak at $1772 \mathrm{~cm}^{-1}$ was assigned to $\mathrm{C}=\mathrm{O}$ stretching (Figure S6e, Movie S11). While the peak at $1409 \mathrm{~cm}^{-1}$ may correspond to core stretching (Figure S6d, Movie S12), this was assigned to $\mathrm{CH}_{2}$ deformation in the alkyl chains denoted as $\delta\left(\mathrm{CH}_{2}\right)$ based on wellestablished literature ${ }^{49-52}$ on alkyl chain vibrations. Along with these peaks, we also tracked the peaks at 1025 and $1063 \mathrm{~cm}^{-1}$ which were assigned to $C-C$ stretching of the trans $v(C-C)_{T}$ and gauche $v(C-C)_{G}$ isomers in the alkyl chains. The alkyl chain peak assignments $\left(\delta\left(\mathrm{CH}_{2}\right), v(\mathrm{C}-\mathrm{C})_{T}, \mathrm{v}(\mathrm{C}-\mathrm{C})_{\mathrm{G}}\right)$ were confirmed based on Raman spectroscopy measured on a similar molecule with shorter side chains (Figure S7). A complete explanation for these assignments can be found in the supplementary information.

To investigate the changes in these vibrational modes during the phase transitions, peaks were fit using OriginPro in both the $1000-1100 \mathrm{~cm}^{-1}$ region and $1350-1600 \mathrm{~cm}^{-1}$ region (Figure $3 \mathrm{~g}$ ). Peak line shape was selected as either Gaussian or Lorentzian based on whether the vibrational mode was associated with the quinoidal core or alkyl chains, respectively. Normally, the line shape in solid crystals is expected to follow a Gaussian curve; however peaks associated with the alkyl chains were fit as Lorentzian due to the disorder, causing more liquid like behavior ${ }^{53-55}$. In the $1000-1600 \mathrm{~cm}^{-1}$ range, which captures the core and alkyl chain vibrations, we note a significant intensity reduction and redshift of the $\delta\left(\mathrm{CH}_{2}\right)$ peak at $1409 \mathrm{~cm}^{-}$ ${ }^{1}$ during the I-II transition. We plotted the intensity ratio with respect to $v(C=C)_{E C C, Q}$ and the peak position 
to show this sudden drop in intensity and redshift of $7 \mathrm{~cm}^{-1}$ in the $\delta\left(\mathrm{CH}_{2}\right)$ at the I-II transition temperature (Figure $\mathbf{3 h}$-i). This trend was repeated across 3 crystals to ensure this change is representative of the I-II transition (Figure $\mathbf{S 8 a , b}$ ), though the exact transition temperature varied by $5{ }^{\circ} \mathrm{C}$ for each crystal due to avalanche behavior. The redshift is indicative of reduced steric hinderance or an increase in the attractive forces between alkyl chains. The intensity decrease, on the other hand, implies alterations to either the orientation or distribution of vibrational states in the alkyl chains. Taken together, these changes show a substantial reorientation of the alkyl chains occurs during the I-II transition.

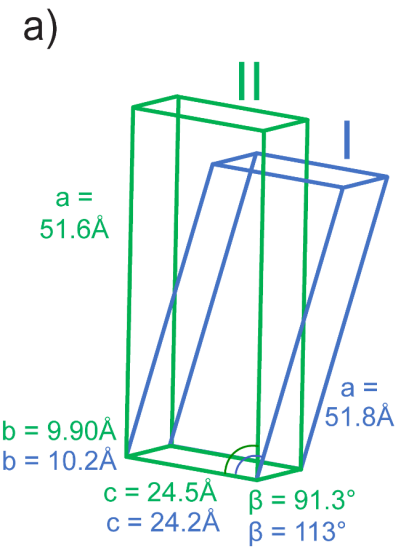

e)

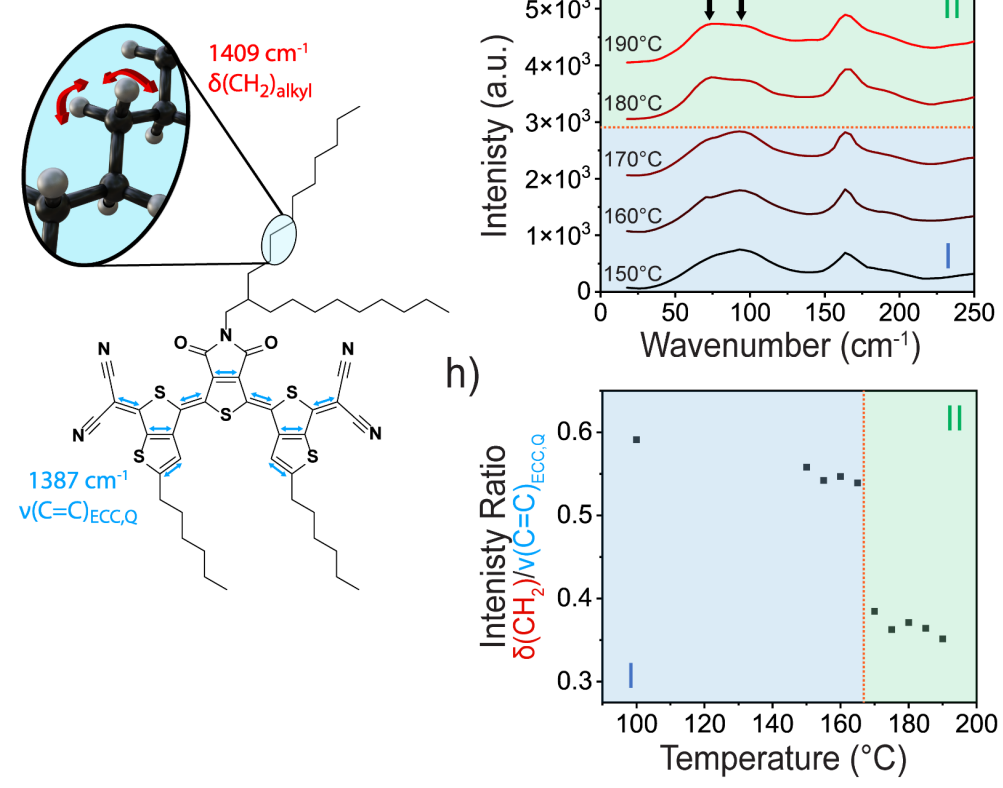

c)

f)
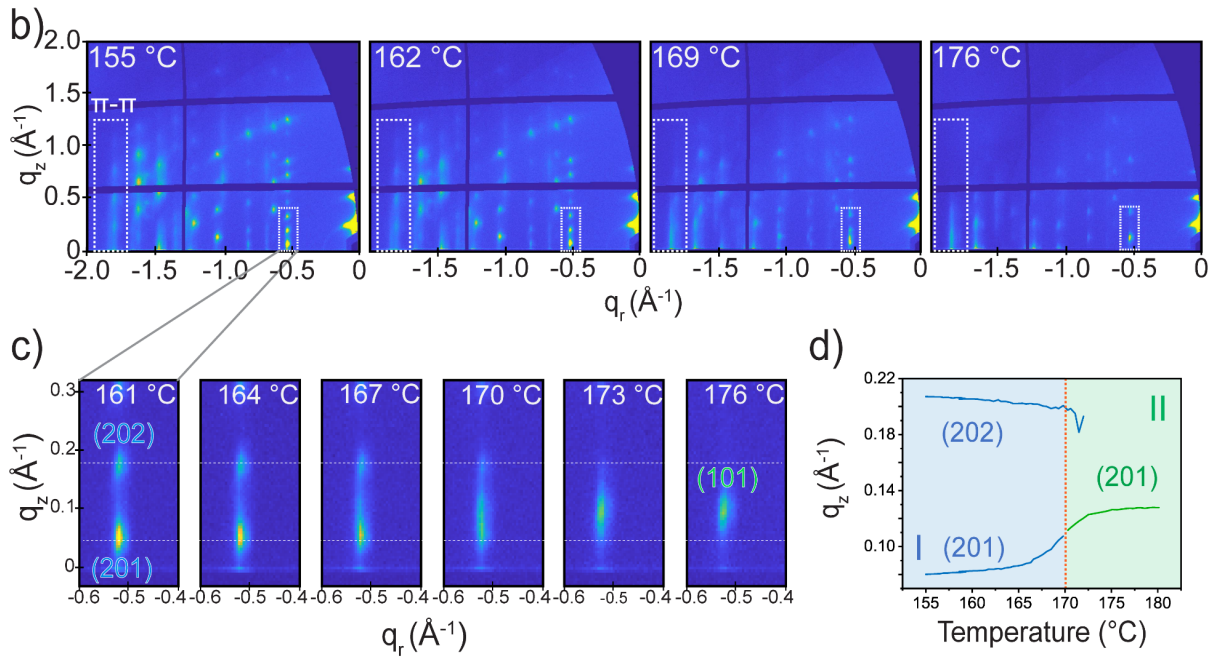

g)

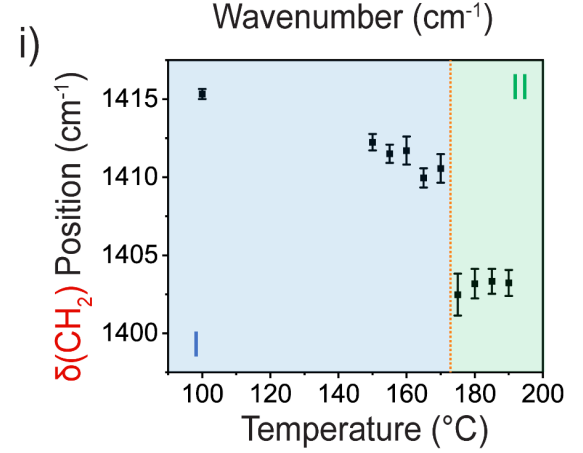

Figure 3. Molecular origins of I-II transition. (a) Unit cell changes from polymorph I (blue) to polymorph II (green). (b) Frames from Movie S7, showing the out of plane peak motion under grazing incidence X-ray diffraction. (c) Changes to the (10) Bragg rod reflecting the change in the $\beta$-angle and (d) associated plot of $\mathrm{q}_{\mathrm{z}}$ peak position during the I-II transition. (e) schematic showing the major vibrational modes associated with the $\mathrm{CH}_{2}$ deformation mode $\left(\delta\left(\mathrm{CH}_{2}\right)\right.$, red $)$ and the $\mathrm{ECC}$ mode for the quinoidal core $\left(\mathrm{v}(\mathrm{C}=\mathrm{C})_{\mathrm{ECC}, \mathrm{a}}\right.$, light blue). Evolution of in situ Raman spectra with increasing temperature for the (f) phonon region and the (g) core $\mathrm{C}=\mathrm{C}$ stretching region. $(\mathrm{h})$ Decrease in the intensity ratio of $\delta\left(\mathrm{CH}_{2}\right)$ to $\mathrm{v}(\mathrm{C}=\mathrm{C})_{\mathrm{ECC}, \mathrm{Q}}$ tracking the 
change in alkyl chain conformation during the I-II phase transition. $(\mathrm{g}) \delta\left(\mathrm{CH}_{2}\right)$ peak position change during the I-II phase transition, which redshifts along with the decrease in intensity. Error bars reflect error in the peak fitting and orange dashed lines indicate transition temperatures.

For the II-III polymorph transition, we see large changes in the unit cell, as polymorph III appears to form a likely hexagonal crystal system (Figure 4a), obtained from the GIXD pattern as discussed in previous work ${ }^{35}$. This is consistent with other previous observations which have shown crystals have a propensity to increase in symmetry at higher temperatures ${ }^{56}$. GIXD video of the II-III transition (Figure 4b, Movie S13) showed the significant reduction in observable peaks, as a result of increased symmetry of the hexagonal lattice as well as reduced long-range order as higher q peaks are no longer measurable (to be discussed later). Despite the reduced diffraction of other Bragg rods at higher q values, the $\pi$-stacking peak remains intense and well defined in polymorph III, in line with a continued dominant $\pi$-stacking motif. The reconstructive transition is further highlighted by observing the complete disappearance of the polymorph II diffraction peaks at the expense of polymorph III peaks (Figure 4c,d), with no shift in position prior to transition, which is consistent with a nucleation and growth type mechanism.

Upon investigation of the II-III transition mechanism via Raman spectroscopy, we observed something quite unexpected: we see the marked weakening of the core stretching peak $v(C=C)_{E C C, Q}$ at $1387 \mathrm{~cm}^{-1}$, while 2 new peaks appear at higher wavenumbers, 1442 and $1497 \mathrm{~cm}^{-1}$ (Figure 4e,f). This indicated a modification to the conjugated core itself. We initially suspected this may be a degradation of the molecule at such high temperatures. However, the temperature at which the peaks appeared were highly correlated with the II-III transition and, moreover, this peak formation was found to be reversible (Figure S9). Along with this, we also observed a change in the intensity ratio between the peaks located at 1025 and $1063 \mathrm{~cm}^{-1}$, previously assigned to the trans $\left(\mathrm{V}(\mathrm{C}-\mathrm{C})_{\mathrm{T}}\right)$ and gauche $\left(\mathrm{V}(\mathrm{C}-\mathrm{C})_{\mathrm{G}}\right)$ isomers, respectively. Based on these observations, we hypothesize a transformation from a quinoidal structure to aromatic structure accompanied by decreased order of the alkyl side chains; such quinoidal to aromatic transitions have been observed in similar systems $s^{12,27,29,57}$.

Previous work on similar quinoidal systems has revealed the presence of a singlet biradical ground state which affects the molecular structure and, by extension, the intermolecular interactions. Moreover, previous work on these biradical forms showed a sensitivity to temperature offering the intriguing possibility that the II-III transition occurred alongside a quinoidal to aromatic transition ${ }^{12,33}$. The key change in molecular structure between a quinoidal and aromatic form is the alternation between the single and double bonds along the conjugated backbone, which is described by the bond length alternation (BLA) parameter, the average difference between consecutive single and double $\mathrm{C}-\mathrm{C}$ bonds. $\mathrm{A}$ $\mathrm{BLA}<0$ defines the quinoidal structure whereas a BLA $>0$ defines the aromatic form. Because of this change in bond length, Raman spectroscopy is quite sensitive to these biradical forms, due to the difference in vibrational frequency of the ECC mode in the quinoidal and aromatic systems ${ }^{12,27,29,57}$. We simulated the Raman spectrum for this scenario by forcing the BLA into the aromatic form via substituting the cyano groups for methyl groups on the ends of the molecules and compared this to the spectra obtained from polymorph III (Figure S10). This allowed us to assign the two new peaks at 1442 and 1497 $\mathrm{cm}^{-1}$ to the ECC of the aromatic form $\mathrm{v}(\mathrm{C}=\mathrm{C})_{\mathrm{ECC}, \mathrm{A}}$ (Figure S10b, Movie S14) and asymmetric stretching modes along the conjugated backbone $\left(\mathrm{v}(\mathrm{C}=\mathrm{C})_{\text {asym }}\right)$ (Figure S10c, Movie S15) respectively. The complete explanation for these assignments is found in the supplementary information. 
Based on this assignment, we plotted the intensity ratio of the $1442 \mathrm{~cm}^{-1}$ to the $1387 \mathrm{~cm}^{-1}$ peaks, representing the ratio of aromatic to quinoidal forms, as we increased temperature (Figure $\mathbf{4 g}$, top). We observe a significant increase in the biradical form starting at the II-III transition. Moreover, the intensity ratio of trans to gauche $\mathrm{C}-\mathrm{C}$ stretching also changed during the transition (Figure $\mathbf{4 g}$, bottom). As polymorph III formed, this ratio decreases drastically, suggesting significant changes in the alkyl chain dynamics. In previous works, the intensity ratio of these two conformers provide an indication of disorder in the alkyl side chains ${ }^{49,51,58,59}$. As more gauche isomers form in the system, the alkyl chains become bent and twisted within the occupied volume. A complete melting of the alkyl side chains is represented by a ratio of 1 , as the chains become free to bend in any direction. In the 2DQTT-o-B system, the intensity ratio of trans to gauche decreases from 8 to 1 across the II-to-III transition. These results strongly suggest that the formation of biradical species and alkyl chain melting are the molecular origins of the II-III phase transition.
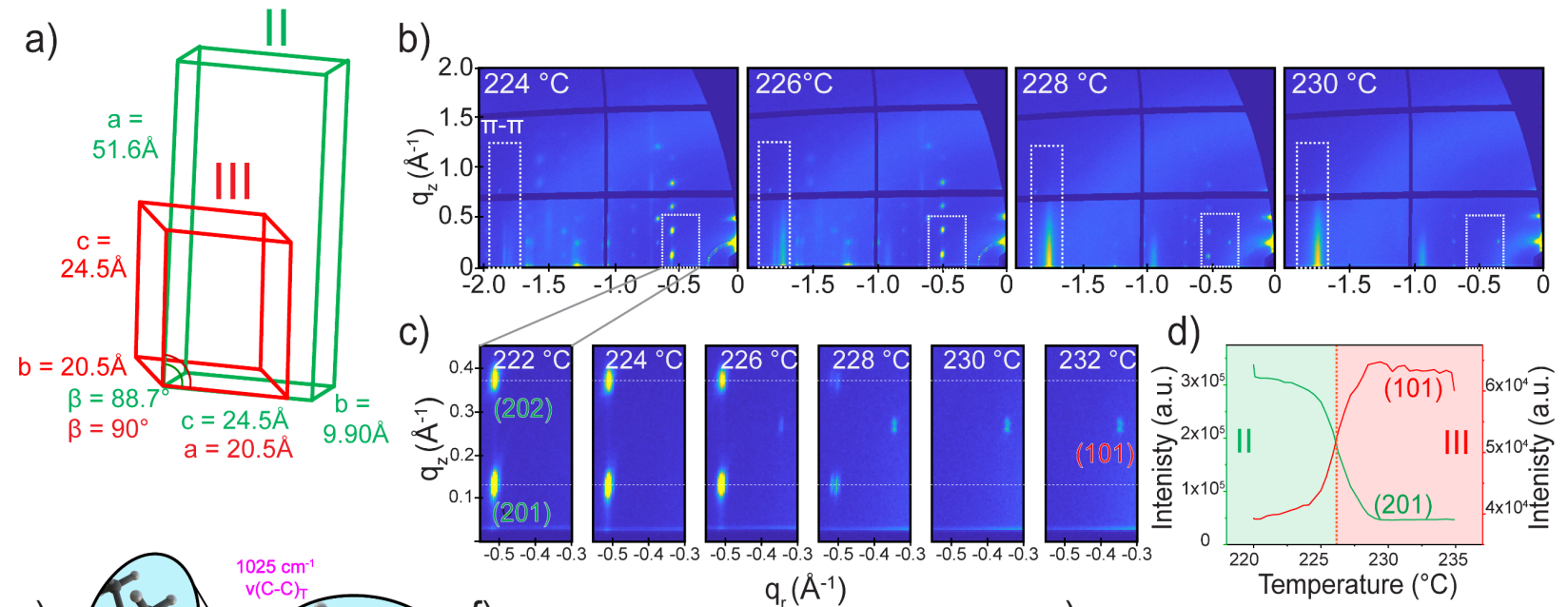

e)

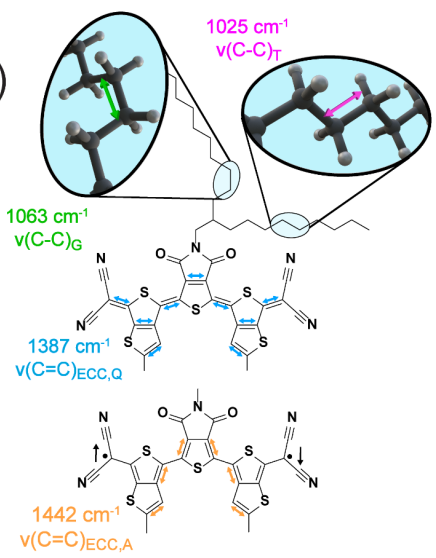

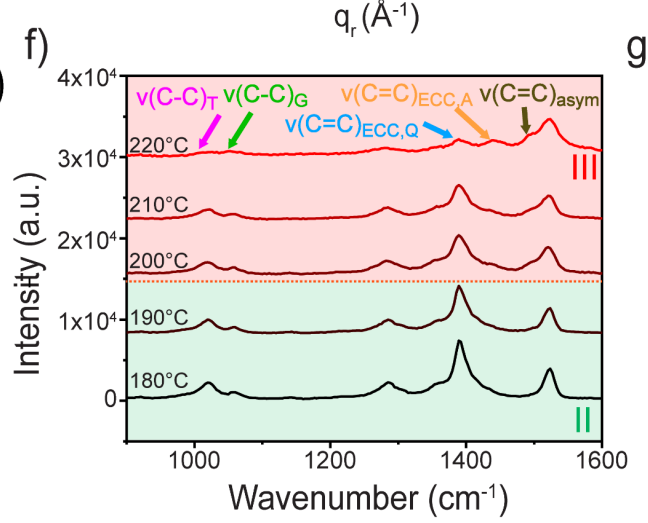

d)

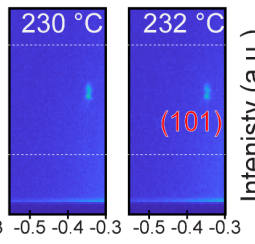

g)
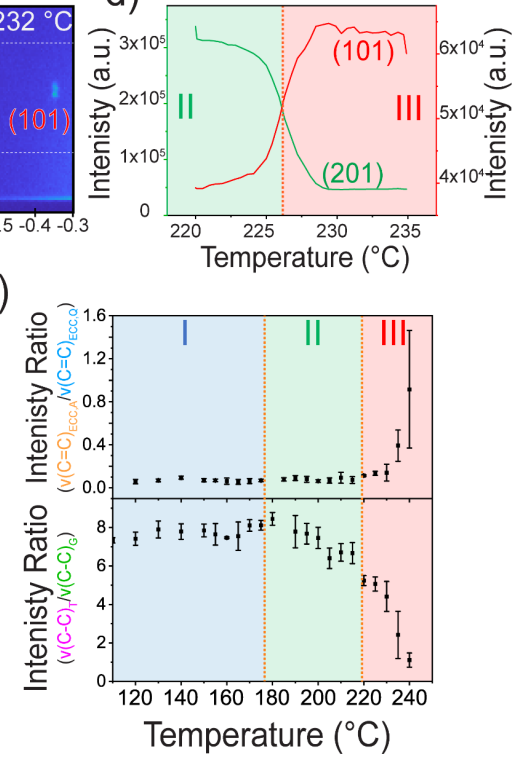

Figure 4. Molecular origins of II-III transition. (a) Unit cell changes from polymorph II (green) to polymorph III (red). (b) Frames from (Movie S13), showing the diffraction peak evolutions during in situ GIXD. (c) Enlarged view of the (20) (polymorph II) and (10) (polymorph III) Bragg rods and (d) associated plot of $q_{z}$ peak position during the I-II transition. (e) Main vibrational modes for the peaks assigned to the

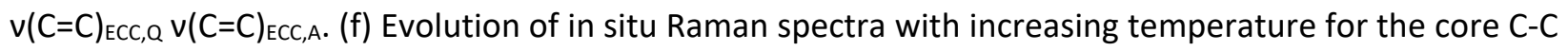
stretching region. (g) Intensity ratio of aromatic conjugated core to quinoidal core increase during II-III phase transition (Top), and corresponding decrease in the trans to gauche intensity ratio (Bottom). 
To confirm the formation of biradicals, we also simulated and compared the absorption spectroscopy as well as measured the spin states in the crystals through in situ EPR spectroscopy to directly observe the formation of these biradical states. UV-Vis was simulated for both the quinoidal and aromatic forms via setting the BLA value for each of the systems (see methods). The simulated UV-Vis spectra for the quinoidal molecule showed good agreement with the experimental spectra (Figure S11a) though we expect the redshift and vibronic fine features resulting from crystalline packing not to be captured in the simulation. For the aromatic molecule, the simulation showed a significantly red shifted and broadened peak compared to the quinoidal form, similar to the redshift observed in the experimental spectra (Figure S11b). Based on Raman, we expect a mixture of quinoidal and aromatic forms, which may account for a smaller extent of red shift observed than predicted by simulation (Figure S11c,d). Nonetheless, the good qualitative match between simulated and experimental spectra suggests that the biradical aromatic form provides a good model for describing the molecular system in polymorph III. Finally, as expected from our hypothesis, in situ EPR showed an increasing presence of spins in our system as we increased temperature (Figure 5a). EPR directly measures the increasing unpaired spins associated with the biradical state (Figure 5a) and indeed, we see a dramatic increase in the concentration of spins within the material (40x increase) upon reaching the II-III transition (Figure 5b). This confirms that the II-III transition not only exhibits a large structural change, but also that a key part of this transition involves accommodating the abruptly increased concentration of biradical species through a reconstructive structural transition (Figure 5c). 
a)
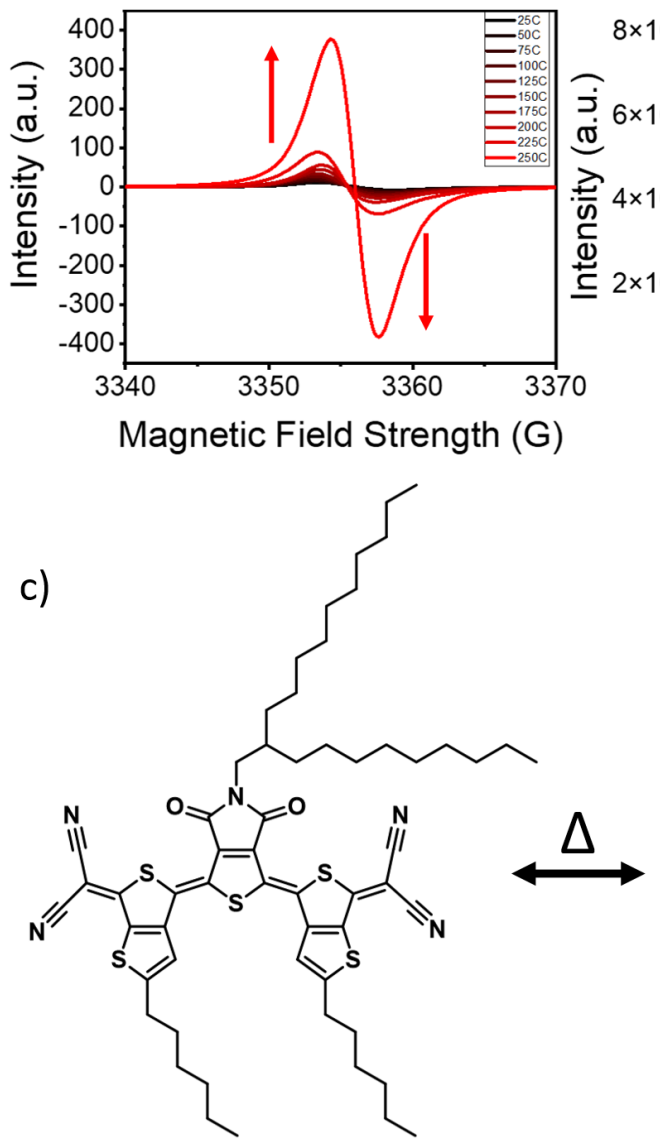

b)
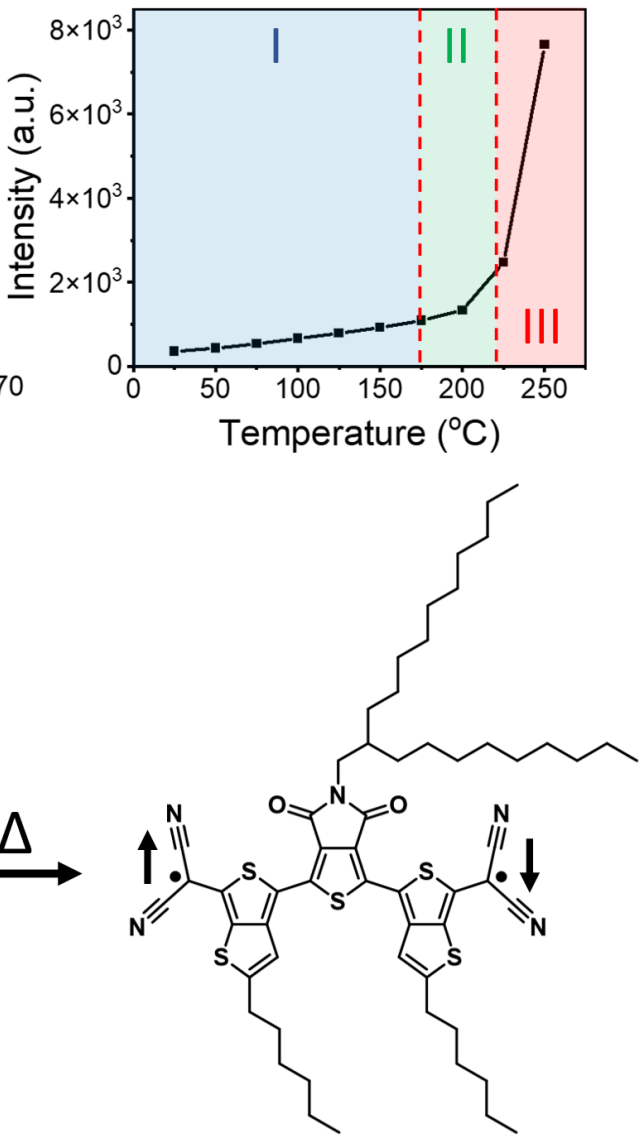

Figure 5. (a) In situ EPR spectra (b) doubly integrated intensity across varying temperature showing a substantial increase in number of spins with heating. (c) Proposed schematic for biradical formation associated with the EPR signal.

Proposed Structural Transition Mechanisms. Based on the molecular changes described previously, we suggest the I-II cooperative transition occurs via a change in the tilt in the molecule driven by alkyl side chain conformation change (Figure 6a). Much like stacks of dominos tilting all at once, molecules along the $b$-axis of the crystal constituting the 1-D $\pi$ stacks stand up during the I-II transition. In this case, the tipping of the molecule is caused by a conformation change in the alkyl side chains, which results in the molecule standing upright during heating and allowing the molecule to slip back into a tilted position during cooling. We suggest engineering of these alkyl side chains may be crucial in facilitating the I-II cooperative behavior. While recently rotation of bulky side chains have been shown to be critical for expressing cooperative behavior in molecular crystals ${ }^{15,16,18}$, cooperative polymorph transition has rarely been studied in molecules with alkyl side chain motifs - a dominant side chain design in organic crystals $^{23,60,61}$. Until now, their role in facilitating cooperative transitions has not been well understood.

The II-III phase transition, however, was shown to be driven by the formation of biradical species and is facilitated by increased mobility of the alkyl side chains during a melting process. At room temperature, 
the concentration of biradical cores is quite low, meaning the potential for spin-spin interaction is quite low. However, as the temperature increases through the II-III transition, the biradical concentration abruptly increases conducive to forming new spin-spin interactions ${ }^{27,33}$. Typically, in molecules where the biradical ground state is dominant, the major packing motifs are either face-on dimer pairs when unhindered ${ }^{30,62}$ or ladder-like stacking ${ }^{31,63}$ when steric hinderance prevents the former. In both cases, the high spin centers (where the radical is most localized) will line up and, in some circumstances, form a pseudo $\sigma$-bond ${ }^{12,30,33,62}$.

One possibility of molecular packing change during II-III transition is shown in Figure $6 \mathbf{b}$, where molecules form dimers with the nearest neighbor in the original polymorph II structure. Since the crystal packs in these 1-D $\pi$-stacks with dimer-like pairs already, this would be a natural progression. While the dimer formation may be plausible, we cannot say with certainty how those dimers then pack to form polymorph III. Regardless of the new core packing motif, to facilitate the II-III also necessitates alkyl chain melting, indicated by increased gauche conformers. Moreover, this side chain melting is consistent with the kinetic trapping observed (Figure 1e and Movie S4). As we observe upon cooling to $37^{\circ} \mathrm{C}$, the alkyl chains return to a frozen state however there appears to be residual biradicals not yet quenched by cooling, owing to a residual lifetime of the biradical state (Figure S6). As a result, polymorph III is trapped and would not return to polymorph I due to freezing the alkyl chains before the conjugated cores rearrange back into polymorph I that may require quenching of biradical species. However, without the full structure of polymorph III, we are left only to postulate possible molecular packings. Nevertheless, we can suggest the nucleation and growth polymorph transition is the result of increased core-core interactions triggered by the biradical formation, and the large packing changes are necessarily facilitated by increased molecular mobility from the melted alkyl side chains. 

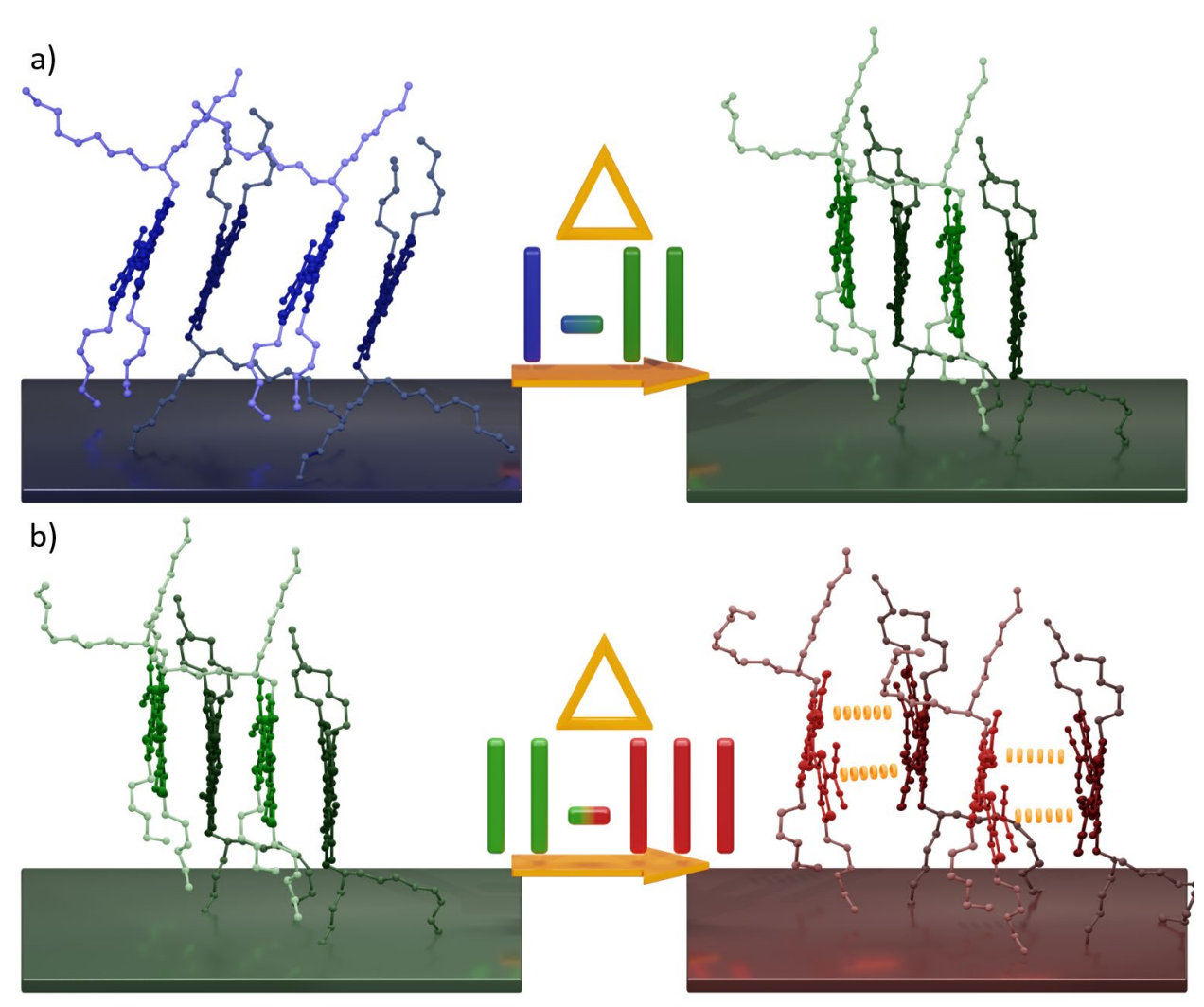

Figure 6. Proposed transition mechanisms for the (a) I-II and (b) II-III transitions.

Electronic and device properties. We worked to demonstrate the control and design of organic electronics utilizing these transition mechanisms. We fabricated single crystal devices to measure the twopoint-probe conductivity of each polymorph (Figure 7a,b). The crystals were pre-bent fixed at two ends by PEDOT:PSS electrodes. When heated to trigger I-II transition, the crystal would straighten from the prebent state due to shape change shown in Figure 2a. We observed a 6-fold decrease in conductivity at the I-Il transition, with good recoverability during cooling albeit with a hysteresis. Moreover, we see a moderate increase in conductivity upon polymorph II-III transition. We suspect this recovery of conductivity may be a result of charge carrier doping through the presence of biradicals, similar to Wudl et. al., which showed a self-doping effect ${ }^{34}$. This self-doping process may compete with the reduction in charge transfer pathways due to structural transitions.

We then were able to harness the shape change discovered in the I-II transition for reliable thermal actuation (Figure 7c,d). For the device, we attached a crystal at both ends using PEDOT:PSS and upon heating, the crystal became dislodged on one side due to the cooperative shape change. This allowed for switching on and off the device by cycling between polymorphs I and II, exhibiting an on/off ratio of 500 and effectively reaching the noise floor for device measurement in the off state. Because the crystal became detached in the polymorph II state, we observed no conductivity and cooling back to polymorph I reforms the contact between the crystal and the PEDOT:PSS, turning the device back on. This provided 
quite reliable cycling for several cycles until out of plane bending prevented stable contact between the PEDOT:PSS contacts.

a)

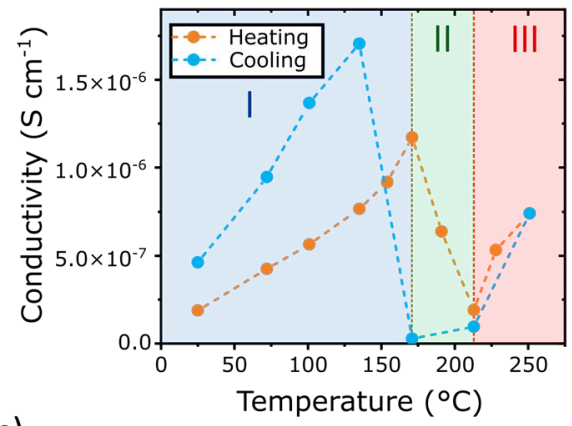

c)

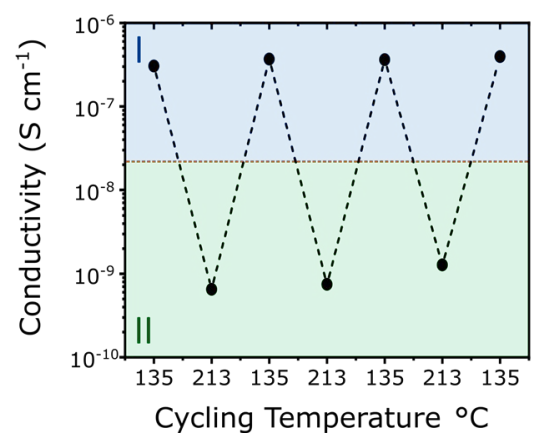

b)

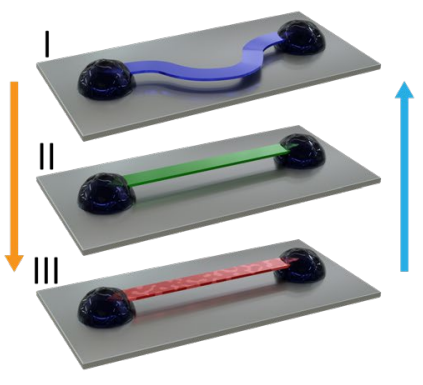

d)

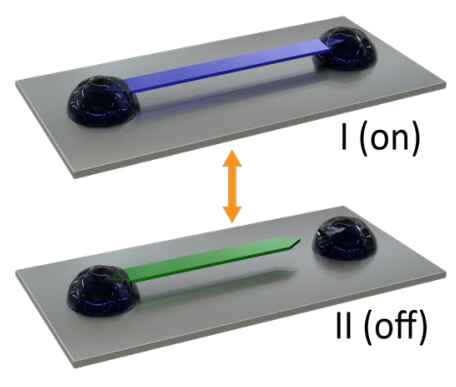

Figure 7. Cooperative transition driven actuator device. (a) in situ temperature variable conductivity measurements showing modulation of the conductance based on polymorph and (b) corresponding device architecture. (c) Cycling of single crystal actuator device and (d) corresponding device architecture.

\section{Conclusions}

We observed both cooperative and nucleation and growth behavior within the 2DQTT-o-B system, allowing for direct comparison of molecular origins of these transitions in a single material. We discovered the I-II transition exhibited cooperative behavior, resulting in a rapid, shape changing transition dominated by avalanches as defects were introduced. The transition was found to be driven by changes in the alkyl side chain confirmation, as revealed by Raman spectroscopy. This was in striking contrast with the slow, smooth II-III transition showing clear nucleation and growth behaviors. Unlike the I-II transition, the II-III transition was driven by changes in spin-spin interactions through biradical formation which is the observation of biradical interactions triggering a polymorphic phase transition.

We then harnessed cooperative behaviors for the design and application of novel organic electronic switching behavior. Not only were we able to tune the conductivity by orders of magnitude via the structural changes, but we could also use the mechanical effects to sever the connection with one of the PEDOT:PSS contacts. By taking advantage of the mechanical effects of the cooperative shape change, we could switch on and off a single crystal device through rapid temperature actuation. This opens the door for fusing the novel mechanical effects of cooperative transitions with device architecture for new functionality. Ultimately, understanding the origin of these transition mechanisms offers pathways to rationally designing organic semiconductors to access cooperative transitions with these exciting properties. 


\section{Materials and Methods}

Materials synthesis. Synthesis of 2DQTT-o-B has been published in Reference $25^{24}$.

Single crystal fabrication. Single crystals of 2DQTT-o-B were fabricated through a dropcasting method. Solutions with concentrations between $10-15 \mathrm{mg} \mathrm{mL}^{-1}$ of 2DQTT-o-B dissolved in a 1:1 mixture of dichlorobenzene and decane were heated to $100{ }^{\circ} \mathrm{C}$. 5-15 $\mu \mathrm{L}$ were dropped onto PTS-treated silicon wafers $\left(\mathrm{SiO}_{2} / \mathrm{Si}\right.$ ) and allowed to dry overnight. For large crystals (SCXRD and Raman experiments), 2DQTTo-B was dissolved in a 1:1 mixture of dichloromethane and ethyl acetate at $1 \mathrm{mg} \mathrm{mL}^{-1}$ in a $30 \mathrm{~mL}$ vial (up to about $15 \mathrm{~mL}$ of solution was used). The solution was then capped with parafilm and 1-5 holes were created in the film using a needle. The vial was then placed in a glove bag under nitrogen atmosphere for slow evaporation over a few weeks. The nitrogen in the glove bag was refilled every other day to prevent oxygen from reacting with the molecule and ensure saturation of solvent vapors did not occur.

Polarized optical microscope. Single crystals grown under the dropcasting procedure were placed on a Nikon H550S with a high-speed camera (Infinity 1) and heating stage (Linkam 402). The chamber was sealed with a magnetic lid and O-ring during heating. The temperature ramp was kept at a constant $5{ }^{\circ} \mathrm{C}$ $\mathrm{min}^{-1}$. Videos were recorded at framerates ranging from 1 to $7.5 \mathrm{fps}$. Video analysis was performed using a python program to obtain an average intensity value of a given crystal at each frame.

Grazing incidence X-ray Diffraction. GIXD was performed at beamline 8-ID-E of the Advanced Photon Source at Argonne National Laboratory ${ }^{64}$. The data were collected at $10.91 \mathrm{keV}$ on a 2D Pilatus $1 \mathrm{M}$ detector. Films of polymorph I were obtained by solution coating from a solution of 2DQTT-o-B dissolved in chloroform and chlorobenzene at $6 \mathrm{mg} \mathrm{mL}^{-1}$ onto $\mathrm{SiO}_{2}$ treated with trichloro(phenyl)silane (PTS). Solution coating was performed at $85{ }^{\circ} \mathrm{C}$ at a blade speed of $0.3 \mathrm{~mm} \mathrm{~s}^{-1}$ and a blade gap of $100 \mu \mathrm{m}$. These films were annealed under nitrogen atmosphere for 30 minutes at $100{ }^{\circ} \mathrm{C}$ to convert the films to polymorph I. In situ thermal annealing measurements were conducted in a He environment with the sample on a commercial thermal stage (Linkam HFSX350-GI), with the temperature ramped at $10^{\circ} \mathrm{C} \mathrm{min}^{-}$ ${ }^{1}$ and the exposure taken after equilibration at the target temperature for approximately $5 \mathrm{~min}$. GIXD videos (Movies $\mathbf{S 7 , 1 3}$ ) were obtained via taking continuous exposures at a constant heating of $5^{\circ} \mathrm{C} \mathrm{min}^{-1}$. GIXSGUI software was used to correct for detector nonuniformity, beam polarization and to reshape the $2 \mathrm{D}$ data into the representation $\mathrm{q}_{\mathrm{z}} \mathrm{vs}_{\mathrm{q}^{6}}{ }^{65}$. The incident angle was set at $0.14^{\circ}$ right above the critical angle for total reflectance of the organic thin film. Unit cells were extracted based on the discussion in the previous paper by Davies and Diao et. al., 2021.

Raman Spectroscopy. A Raman confocal imaging microscope with a $532 \mathrm{~nm}$ laser (Laser Quantum Ventus 532 with max power $50 \mathrm{~mW}$ ) and 50x long working distance objective lens (HORIBA LabRAM HR 3D) equipped with HORIBA Synapse back-illuminated deep-depletion CCD camera was used to collect spectra. Using a $300 \mathrm{~g} \mathrm{~mm}^{-1}$ grating, we used a scan exposure time of between 20-60 s. An optical density filter of $O D=0.2$ was used $(O D=\log ($ power transmission factor $))$, and no beam damage was observed only at the highest temperatures after prolonged periods of laser exposure (>5min). To eliminate this effect, each spectrum was recorded in a new position on the crystal to prevent overexposure of any particular area. For variable-temperature experiments, the samples were collected using a Linkam THMS600 heating stage with a closed chamber. The heating and cooling rate was kept at $10^{\circ} \mathrm{C} \mathrm{min}^{-1}$. Each temperature was 
equilibrated until the temperature reading stabilized and the Raman laser was refocused to account for substrate thermal expansion (approximately 5 minutes).

Electron Paramagnetic Resonance Spectroscopy. Crystals of Polymorph I were grown via the slow evaporation method from DCM and EA described in the crystal fabrication above, were dried out and then placed in an EPR tube. The crystal sample was then measured on an EMXplus running the Xenon software with variable temperature (part no. E0000071) for in situ EPR measurements. The temperature was stepped by $25^{\circ} \mathrm{C}$ up through the II-III phase transition. The Intensity at each temperature was then doubly integrated and plotted vs temperature.

Electronic properties. For single crystal devices, large needle like crystals were grown from a $1 \mathrm{mg} \mathrm{mL}^{-1}$ solution of 1:1 mixture of dichloromethane and ethyl acetate. These crystals were pre-bent and attached to a glass substrate, using conductive PEDOT:PSS. Pre-bending allowed for the devices to be heated through the I-II transition without cracking or breaking the device. The device was measured in air using a Keysight B1500A semiconductor parameter analyzer. The current through the crystal was measured by contacting the PEDOT:PSS with 2 probes and current was obtained as a function of source-drain voltage.

Actuator devices were fabricated by attaching the crystal with PEDOT:PSS without pre-bending the crystal. During the first cycle of the I-II transition, one end becomes detached from the PEDOT:PSS due to the crystal shape change. This device can then be cycled multiple times as once cooled down, the crystal reconnects with the PEDOT:PSS contact.

DFT calculations. Ground-state geometries of isolated molecules were optimized at the DFT level using the $\omega B$ 97X-D functional and def2TZVPP basis set. IR and Raman frequencies were calculated at the same level of theory. For the quinoidal form, the original molecule was used, however for the aromatic form, the cyano-groups were cleaved. This allowed for fully relaxed structures to be used in both simulations while capturing the correct bond length alternation observed in each conjugated core. The UV-Vis absorption spectra were likewise calculated at the same level of theory, using the 10 lowest singlet and triplet states in the TD-DFT calculation. All calculations were performed using Gaussian16 Rev. B. Side chains were removed for computational efficiency. In this case, the quinoidal geometry optimization was allowed to relax fully, while for the aromatic geometry the carbon atoms along the backbone were fixed to enforce the proper aromatic single-double bond alternation pattern (i.e., that opposite the quinoidal bonding pattern) and the result of the structure was allowed to relax.

\section{Acknowledgements}

Y.D., D.D. S.K.P. H.C. acknowledge the Sloan Foundation for a Sloan Research Fellowship in Chemistry and a 3M Nontenured Faculty Award. D.D. acknowledges support of DuPont Graduate Fellowship and A. T. Widiger Chemical Engineering Fellowship. Y.D. and P.K. acknowledge partial support by the NSF MRSEC: Illinois Materials Research Center under grant number DMR-1720633 and NSF CAREER award under Grant No. 18-47828. X.Z. acknowledges the National Key R\&D Program of China (2017YFA0204700). This work was conducted in part in the Frederick Seitz Materials Research Laboratory Central Facilities. Portions of this research were carried out at the Advanced Photon Source, a U.S. Department of Energy (DOE), Office of Science User Facility, operated for the DOE Office of Science by Argonne National Laboratory under Contract No. DE-AC02-06CH11357. D.D. acknowledges Dr. Joseph G. Manion (CGFigures) for his tutorials on using 3D rending software for scientific illustrations. 


\section{Author Contributions}

D.W.D. designed and conducted the majority of the experiments and writing of the paper. Y.D. supervised the project and guided the manuscript writing. S.K.P. fabricated and measured the single crystal devices and assisted with related discussions. D.Y. and X.Z. Synthesized the 2DQTT-o-B material. S.S. and B.S. performed the DFT calculations for the Raman and UV-Vis studies. J.S. and P.K. assisted with setup and collection of the GIXD videos. R.W. performed the collection and helped with the analysis for the high temperature EPR spectroscopy.

\section{Additional Information}

Supplementary information is available online. Correspondence and requests for materials should be addressed to Y.D.

\section{Competing Financial Interests}

The authors declare no competing financial interests.

\section{References}

1. Anderson, P. W. More Is Different. Science 177, 393-396 (1972).

2. Whitty, A. Cooperativity and biological complexity. Nature chemical biology 4, 435-9 (2008).

3. van den Ende, J. a., Ensing, B. \& Cuppen, H. M. Energy barriers and mechanisms in solidsolid polymorphic transitions exhibiting cooperative motion. CrystEngComm 18, 4420-4430 (2016).

4. Pérez-Reche, F. J., Vives, E., Mañosa, L. \& Planes, A. Athermal Character of Structural Phase Transitions. Physical Review Letters 87, 195701-195701 (2001).

5. Vesely, S. L., Dolci, S. R., Vesely, A. A. \& Dolci, C. A. Cooperativity as an Emergent Phenomenon. in 2019 IEEE Radio and Antenna Days of the Indian Ocean (RADIO) 1-2 (2019).

6. Commins, P., Desta, I. T., Karothu, D. P., Panda, M. K. \& Naumov, P. Crystals on the move: mechanical effects in dynamic solids. Chem. Commun. 52, 13941-13954 (2016). 
7. Karothu, D. P., Weston, J., Desta, I. T. \& Naumov, P. Shape-Memory and Self-Healing Effects in Mechanosalient Molecular Crystals. J. Am. Chem. Soc. 138, 13298-13306 (2016).

8. Panda, M. K. et al. Colossal positive and negative thermal expansion and thermosalient effect in a pentamorphic organometallic martensite. Nature Communications 5, 4811-4811 (2014).

9. Khalil, A., Ahmed, E. \& Naumov, P. Metal-coated thermosalient crystals as electrical fuses. Chemical Communications 53, 8470-8473 (2017).

10. Takamizawa, S. \& Takasaki, Y. Shape-memory effect in an organosuperelastic crystal. Chem. Sci. 7, 1527-1534 (2016).

11. Mañosa, L. et al. Acoustic emission field during thermoelastic martensitic transformations. Applied Physics Letters 54, 2574-2576 (1989).

12. Yang, K. et al. Stable Organic Diradicals Based on Fused Quinoidal Oligothiophene Imides with High Electrical Conductivity. Journal of the American Chemical Society 142, 4329$4340(2020)$.

13. Bi, D., Henkes, S., Daniels, K. E. \& Chakraborty, B. The Statistical Physics of Athermal Materials. Annu. Rev. Condens. Matter Phys 6, 63-83 (2015).

14. Tuszynski, J. A. et al. Jerky phase-front motion in $\mathrm{PbTiO} 3$ crystals Comments on phasefront propagation in ferroelectrics Athermal martensitic behaviour enhanced in sodium niobate by Mn dopant and axial compression The influence of magnetic fields on the interphase boundary dyn. J. Phys 21, 1257-1263 (1988).

15. Chung, H. et al. Rotator side chains trigger cooperative transition for shape and function memory effect in organic semiconductors. Nature Communications 9, 278-278 (2018). 
16. Chung, H. et al. Understanding the Role of Bulky Side Chains on Polymorphism of BTBTBased Organic Semiconductors. Crystal Growth \& Design 20, 1646-1654 (2020).

17. Diao, Y. et al. Understanding polymorphism in organic semiconductor thin films through nanoconfinement. Journal of the American Chemical Society 136, 17046-17057 (2014).

18. Park, S. K. et al. Super- and Ferroelastic Organic Semiconductors for Ultraflexible SingleCrystal Electronics. Angewandte Chemie International Edition 59, 13004-13012 (2020).

19. Chung, H. et al. Single Atom Substitution Alters the Polymorphic Transition Mechanism in Organic Electronic Crystals. Chemistry of Materials 31, 9115-9126 (2019).

20. Panzer, F., Bässler, H. \& Köhler, A. Temperature Induced Order-Disorder Transition in Solutions of Conjugated Polymers Probed by Optical Spectroscopy. Journal of Physical Chemistry Letters 8, 114-125 (2017).

21. Su, S.-Q. et al. Assembling an alkyl rotor to access abrupt and reversible crystalline deformation of a cobalt(II) complex. Nature Communications 6, 8810-8810 (2015).

22. Sato, O. Dynamic molecular crystals with switchable physical properties. Nature Chemistry 8, 644-656 (2016).

23. Dharmarwardana, M. et al. Rapidly Reversible Organic Crystalline Switch for Conversion of Heat into Mechanical Energy. J. Am. Chem. Soc. 143, 5951-5957 (2021).

24. Zhang, C. et al. Pursuing High-Mobility n-Type Organic Semiconductors by Combination of “Molecule-Framework" and "Side-Chain" Engineering. Advanced Materials 28, 8456-8462 (2016).

25. Zhao, Y., Guo, Y. \& Liu, Y. 25th Anniversary Article: Recent Advances in n-Type and Ambipolar Organic Field-Effect Transistors. Advanced Materials 25, 5372-5391 (2013). 
26. Suzuki, Y., Miyazaki, E. \& Takimiya, K. ((Alkyloxy)carbonyl)cyanomethylene-Substituted Thienoquinoidal Compounds: a New Class of Soluble n-Channel Organic Semiconductors for Air-Stable Organic Field-Effect Transistors. Journal of the American Chemical Society 132, 10453-10466 (2010).

27. Yuan, D. et al. Cholesteric Aggregation at the Quinoidal-to-Diradical Border Enabled Stable n-Doped Conductor. Chem 5, 964-976 (2019).

28. Yuan, D. et al. Air-Stable n-Type Thermoelectric Materials Enabled by Organic Diradicaloids. Angewandte Chemie International Edition 58, 4958-4962 (2019).

29. Ortiz, R. P. et al. Quinoidal Oligothiophenes: Towards Biradical Ground-State Species. Chemistry - A European Journal 16, 470-484 (2010).

30. Zafra, J. L. et al. Reversible Dimerization and Polymerization of a Janus Diradical To Produce Labile $\mathrm{C}-\mathrm{C}$ Bonds and Large Chromic Effects. Angewandte Chemie International Edition 55, 14563-14568 (2016).

31. Shimizu, A. et al. Resonance Balance Shift in Stacks of Delocalized Singlet Biradicals. Angewandte Chemie International Edition 48, 5482-5486 (2009).

32. Kubo, T. et al. Singlet Biradical Character of Phenalenyl-Based Kekulé Hydrocarbon with Naphthoquinoid Structure. Organic Letters 9, 81-84 (2006).

33. Mou, Z. \& Kertesz, M. Pancake Bond Orders of a Series of $\pi$-Stacked Triangulene Radicals. Angewandte Chemie (International ed. in English) 56, 10188-10191 (2017).

34. Zhang, Y. et al. Temperature Tunable Self-Doping in Stable Diradicaloid Thin-Film Devices. Advanced Materials 27, 7412-7419 (2015).

35. Davies, D. W. et al. Radically Tunable n-Type Organic Semiconductor via Polymorph Control. Chemistry of Materials 33, 2466-2477 (2021). 
36. Chung, H., Ruzié, C., Geerts, Y. \& Diao, Y. Hybrid Mechanism of Nucleation and Cooperative Propagation in a Single-Crystal-to-Single-Crystal Transition of a Molecular Crystal. Crystal Growth \& Design 18, 4245-4251 (2018).

37. Planes, A., Mañosa, L. \& Vives, E. Acoustic emission in martensitic transformations. Journal of Alloys and Compounds 577, S699-S704 (2013).

38. Park, S. K. \& Diao, Y. Martensitic transition in molecular crystals for dynamic functional materials. Chem. Soc. Rev. 49, 8287-8314 (2020).

39. Panda, M. K. et al. Strong and Anomalous Thermal Expansion Precedes the Thermosalient Effect in Dynamic Molecular Crystals. Sci Rep 6, 29610 (2016).

40. Fleury, P. A. Phase Transitions, Critical Phenomena, and Instabilities. Science 211, 125-131 (1981).

41. Cook, H. E., Suezawa, M., Kajitani, T. \& Rivaud, L. Pretransition Phenomena. J. Phys. Colloques 38, C7-439 (1977).

42. Tandon, P., Förster, G., Neubert, R. \& Wartewig, S. Phase transitions in oleic acid as studied by X-ray diffraction and FT-Raman spectroscopy. Journal of Molecular Structure 524, 201215 (2000).

43. Wood, S. et al. Precise Characterisation of Molecular Orientation in a Single Crystal FieldEffect Transistor Using Polarised Raman Spectroscopy. Sci Rep 6, 33057 (2016).

44. Pan, M. et al. Raman study of the phase transition in VO2 thin films. Journal of Crystal Growth 268, 178-183 (2004).

45. Mintairov, A. M., Merz, J. L., Vlasov, A. S. \& Vinokurov, D. V. Observation of a martensitic transition in the Raman spectra of spontaneously ordered GaInP alloys. Semicond. Sci. Technol. 13, 1140-1147 (1998). 
46. Casado, J., Ponce Ortiz, R. \& López Navarrete, J. T. Quinoidal oligothiophenes: new properties behind an unconventional electronic structure. Chemical Society Reviews 41, $5672-5686(2012)$.

47. Castiglioni, C., Lopez Navarrete, J. T., Zerbi, G. \& Gussoni, M. A simple interpretation of the vibrational spectra of undoped, doped and photoexcited polyacetylene: Amplitude mode theory in the GF formalism. Solid State Communications 65, 625-630 (1988).

48. Ferrari, A. C., Robertson, J., Castiglioni, C., Tommasini, M. \& Zerbi, G. Raman spectroscopy of polyconjugated molecules and materials: confinement effect in one and two dimensions. Philosophical Transactions of the Royal Society of London. Series A: Mathematical, Physical and Engineering Sciences 362, 2425-2459 (2004).

49. Venkataraman, N. V., Bhagyalakshmi, S., Vasudevan, S. \& Seshadri, R. Conformation and orientation of alkyl chains in the layered organic-inorganic hybrids: $(\mathrm{CnH} 2 \mathrm{n}+1 \mathrm{NH} 3) 2 \mathrm{PbI} 4$ ( $\mathrm{n}=12,16,18)$. Physical Chemistry Chemical Physics 4, 4533-4538 (2002).

50. G. Brown, K., Bicknell-Brown, Ellen. \& Ladjadj, Meriem. Raman-active bands sensitive to motion and conformation at the chain termini and backbones of alkanes and lipids. The Journal of Physical Chemistry 91, 3436-3442 (2002).

51. J. Orendorff, C., W. Ducey, M. \& E. Pemberton, J. Quantitative Correlation of Raman Spectral Indicators in Determining Conformational Order in Alkyl Chains. The Journal of Physical Chemistry A 106, 6991-6998 (2002).

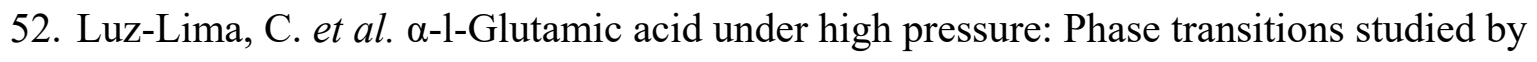
Raman spectroscopy. Vibrational Spectroscopy 86, 343-349 (2016).

53. Pelikan, P., Ceppan, M. \& Liska, M. Applications of numerical methods in molecular spectroscopy. Chap. 1 (CRC Press, 1993). 
54. Schweizer, K. S. \& Chandler, D. Vibrational dephasing and frequency shifts of polyatomic molecules in solution. J. Chem. Phys. 76, 2296-2314 (1982).

55. Bradley, M. S. \& Krech, J. H. High-pressure Raman spectra of the acetone carbonyl stretch in acetone-methanol mixtures. J. Phys. Chem. 97, 575-580 (1993).

56. Filatov, S. K. General concept of increasing crystal symmetry with an increase in temperature. Crystallography Reports 56, 953-961 (2011).

57. Casado, J., Ortiz, R. P. \& Navarrete, J. T. L. Quinoidal oligothiophenes: new properties behind an unconventional electronic structure. Chem. Soc. Rev. 41, 5672-5686 (2012).

58. Kotula, A. P., Walker, A. R. H. \& Migler, K. B. Raman analysis of bond conformations in the rotator state and premelting of normal alkanes. Soft Matter 12, 5002-5010 (2016).

59. Jin, Y., Kotula, A. P., Walker, A. R. H., Migler, K. B. \& Lee, Y. J. Phase-specific Raman analysis of n-alkane melting by moving-window two-dimensional correlation spectroscopy. Journal of Raman Spectroscopy 47, 1375-1384 (2016).

60. Smets, M. M. H. et al. On the mechanism of solid-state phase transitions in molecular crystals - the role of cooperative motion in (quasi)racemic linear amino acids. IUCrJ 7, 331$341(2020)$.

61. Smets, M. M. H. et al. Understanding the Solid-State Phase Transitions of dl-Norleucine: An in Situ DSC, Microscopy, and Solid-State NMR Study. Crystal Growth \& Design 15, 51575167 (2015).

62. Brown, J. T., Zeller, M. \& Rosokha, S. V. Effects of structural variations on $\pi$-dimer formation: long-distance multicenter bonding of cation-radicals of tetrathiafulvalene analogues. Phys. Chem. Chem. Phys. 22, 25054-25065 (2020). 
63. Wehrmann, C. M., Charlton, R. T. \& Chen, M. S. A Concise Synthetic Strategy for Accessing Ambient Stable Bisphenalenyls toward Achieving Electroactive Open-Shell $\pi$ Conjugated Materials. J. Am. Chem. Soc. 141, 3240-3248 (2019).

64. Jiang, Z. et al. The dedicated high-resolution grazing-incidence X-ray scattering beamline 8ID-E at the Advanced Photon Source. Journal of Synchrotron Radiation 19, 627-636 (2012).

65. Jiang, Z. GIXSGUI: a MATLAB toolbox for grazing-incidence X-ray scattering data visualization and reduction, and indexing of buried three-dimensional periodic nanostructured films. Journal of Applied Crystallography 48, 917-926 (2015). 